\title{
Short communication: Antimicrobial susceptibility profiling and genotyping of Staphylococcus aureus isolates from bovine mastitis in Poland
}

\author{
T. Jagielski, ${ }^{1}$ E. Puacz,† A. Lisowski,ł P. Siedlecki,§ W. Dudziak,\# J. Międzobrodzki,\# and H. Krukowskił \\ *Department of Applied Microbiology, Institute of Microbiology, Faculty of Biology, University of Warsaw, 02-096 Warsaw, Poland \\ †National Chamber of Medical Laboratory Specialists, 03-428 Warsaw, Poland \\ †Department of Animal and Environmental Hygiene, University of Life Sciences, 20-950 Lublin, Poland \\ $\S$ Department of Plant Molecular Biology, Institute of Plant Experimental Biology and Biotechnology, Faculty of Biology, University of Warsaw, \\ 02-096 Warsaw, Poland \\ \#Department of Microbiology, Faculty of Biochemistry, Biophysics and Biotechnology, Jagiellonian University, 30-387 Cracow, Poland
}

\begin{abstract}
Staphylococcus aureus is the predominant causative agent of bovine mastitis, a disease that remains a major economic burden for the dairy industry worldwide. In this study, the antimicrobial resistance patterns and the genetic composition of $80 \mathrm{~S}$. aureus mastitis isolates collected from 14 dairy farms in Eastern Poland were determined. Of the 10 antimicrobial agents evaluated, only testing for penicillin $\mathrm{G}$ produced drug resistance. As $41 \%$ of the S. aureus isolates were penicillin resistant, this drug along with other $\beta$-lactamase-sensitive $\beta$-lactams, should rather not be considered for the treatment of bovine mastitis caused by $S$. aureus. Upon genotyping, with a triplex PCR method, a total of 11 distinct PCR types were produced. The population structure of $S$. aureus isolates was highly clonal, with 1 predominant genotype circulating on each farm. The observed similarities in the genotype composition of $S$. aureus populations from geographically distant farms underscore the significance of interfarm transmission of $S$. aureus in Poland. This, in turn, argues for the establishment of a nationwide surveillance program for bovine mastitis due to this pathogen.
\end{abstract}

Key words: Staphylococcus aureus, bovine mastitis, drug resistance, genotyping

\section{Short Communication}

Bovine mastitis persists as the most prevalent disease of dairy herds worldwide and represents one of the most significant economic challenges for the global dairy industry. The total economic burden from mastitis, both clinical and subclinical, has been calculated at up to

Received May 4, 2014.

Accepted June 24, 2014.

${ }^{1}$ Corresponding author: t.jagielski@biol.uw.edu.pl
$€ 200$ per cow yearly (Huijps et al., 2008). The losses are mainly due to a drastically decreased milk production and also due to the costs of veterinary treatment, early culling, and replacements. A wide spectrum of microorganisms has been implicated in the causation of bovine mastitis, including bacteria, viruses, fungi, and algae (Krukowski et al., 2006; Malinowski et al., 2006; Miranda-Morales et al., 2008; Tenhagen et al., 2009; Botrel et al., 2010; Persson et al., 2011). Yet, the vast majority of bovine mastitis cases are of bacterial etiology, with only a few species predominating. Among these, Staphylococcus aureus ranks first, producing prevalence rates of 10 to $30 \%$ in mastitis-affected cattle (Malinowski et al., 2006; Miranda-Morales et al., 2008; Tenhagen et al., 2009; Botrel et al., 2010; Persson et al., 2011). Staphylococcus aureus mastitis presents with varying degrees of severity, ranging from mild and selflimiting to rapidly progressing and fatal. However, the disease most often presents in a subclinical form, which usually evolves into chronic status, characterized by extensive fibrosis, microabscess formation, and occlusion of the ducts and alveoli of the secretory tissue (Zadoks et al., 2011). Of particular concern is that $S$. aureus mastitis infections are often refractory to standard intramammary treatment regimens; one reason for this is increased resistance of the staphylococci to different antimicrobial agents. Indeed, several studies have reported on $S$. aureus mastitis isolates resistant, at different levels, to a wide array of commonly used antibiotics (Malinowski et al., 2002; Moroni et al., 2006; Sakwinska et al., 2011b; Gao et al., 2012). Monitoring of drug resistance in $S$. aureus from dairy herds has been considered as an essential part of mastitis control strategies, as it guides the selection of the most appropriate and efficient therapy for staphylococcal mammary infections.

The advent of molecular typing methods has considerably contributed to the understanding of the epidemiology of many human and animal pathogens. Genotyping has also become an important clinical tool 
to investigate outbreaks, infection sources, and transmission routes of bovine mastitis caused by $S$. aureus. Recently, an increasing amount of research has been conducted into the molecular epidemiology of $S$. aureus mammary infections (Jørgensen et al., 2005; Peles et al., 2007; Ikawaty et al., 2009; Sakwinska et al., 2011b; Gao et al., 2012). However, molecular data on isolates from Poland are still relatively scarce. The purpose of this study was to determine the antimicrobial resistance patterns and the genetic composition of $S$. aureus mastitis isolates collected from dairy herds in Poland.

\section{Study Design and Sampling}

The study was conducted on 14 dairy farms (I-XIV) located in Lublin Province, Eastern Poland, over a 3-yr period (2007-2009). The herds comprised between 12 and 168 cows (median herd size of 33 cows) of the Polish Holstein-Friesian breed and raised under similar conditions of maintenance, feeding, and veterinary care. Throughout the study period, a total of 815 milk samples from 419 cows (out of 673 examined) with clinical and subclinical mastitis were tested. The diagnosis of mastitis was made on the basis of the California mastitis test, clinical examination of the udder, and microbiological examination of milk samples. The milk samples were collected aseptically into sterile glass vials from all quarters that were California mastitis test positive or upon clinical examination, or both. All milk samples for microbiological culturing were collected before milking. The surface of the teat ends was disinfected by wiping it with clean cotton dipped in $95 \%$ alcohol. The samples were cooled and immediately transported to the laboratory.

\section{Isolation and Identification of S. aureus}

Equal volumes $(0.1 \mathrm{~mL})$ of milk samples were inoculated onto $5 \%$ Columbia sheep blood agar (Merck KGaA, Darmstadt, Germany) plates and incubated at $37^{\circ} \mathrm{C}$ for 24 to $48 \mathrm{~h}$. Colonies presumptive of staphylococci based on morphology, hemolysis, and Gram staining, were further tested for catalase, coagulase, and clumping factor production (Slidex Staph Plus; bioMérieux SA, Marcy-l'Étoile, France). Final species identification was achieved by using 2 commercially available biochemical identification systems: STAPHYtest 24 (Pliva-Lachema AS, Brno, Czech Republic) and Phoenix (Becton, Dickinson and Co., Franklin Lakes, NJ). Species assignment was valid if the identification results were congruent and the confidence levels of identification equaled or exceeded $98 \%$ in both systems.

\section{Antimicrobial Susceptibility Profiling}

Antimicrobial susceptibility testing was performed on Mueller-Hinton agar (Oxoid Ltd., Basingstoke, UK) by the disc diffusion method, following the recommendations of the Clinical Laboratory Standards Institute (CLSI, 2012). The antimicrobial agents tested included penicillin $(10 \mathrm{U})$, erythromycin $(15 \mu \mathrm{g})$, clindamycin $(2 \mu \mathrm{g})$, gentamicin $(10 \mu \mathrm{g})$, doxycycline $(30 \mu \mathrm{g})$, ciprofloxacin $(5 \mu \mathrm{g})$, chloramphenicol $(30 \mu \mathrm{g})$, sulfamethoxazole/trimethoprim $(23.75 / 1.25 \mu \mathrm{g})$, and cefoxitin (30 $\mu \mathrm{g})$ as a surrogate marker for methicillin resistance. In addition, susceptibility to vancomycin was determined with E-test stripes (AB Biodisk, Solna, Sweden) in accordance with the manufacturer's instructions. Isolates were categorized as susceptible, intermediate, or resistant, under the Clinical Laboratory Standards Institute breakpoints (CLSI, 2012). The reference strain S. aureus ATCC 25923 served as quality control for the disc diffusion method, whereas vancomycin-susceptible Enterococcus faecalis ATCC 29212 and vancomycinresistant E. faecalis ATCC 51299 strains were used to validate the performance of the Epsilometer test (Etest) assay.

\section{Preparation of DNA}

The bacterial genomic DNA was extracted using the Genomic DNA Prep Plus kit (A\&A Biotechnology, Gdynia, Poland), according to the protocol provided by the manufacturer. The purified DNA was quantified with a NanoDrop ND-1000 spectrophotometer (NanoDrop Technologies Inc., Wilmington, DE) and stored at $-20^{\circ} \mathrm{C}$ until used.

\section{Genotyping}

Genotyping was carried out with a triplex PCR coamplifying 3 loci within the $S$. aureus genome; that is, part of the coa gene coding for coagulase, part of the spa gene coding for protein $\mathrm{A}$, and part of the hypervariable region adjacent to the mecA gene, essentially as described elsewhere (Strandén et al., 2003). The DNA fingerprints were analyzed with the PyElph software (version 1.4; source code available from http://sourceforge.net/projects/pyelph/; Pavel and Vasile, 2012). A dendrogram was constructed by the unweighted pair group method with arithmetic averages (UPGMA) algorithm, with a band position tolerance of $5 \%$. A cluster was defined as 2 or more isolates exhibiting $100 \%$ identity of their triplex PCR patterns, whereas those nonclustered were referred to as unique. Clusters were 
designated after the respective PCR patterns, with Latin uppercase letters.

One hundred two (12.5\%) of the quarter milk samples yielded $S$. aureus cultures, as evidenced on initial screening (positivity in both slide and tube coagulase tests). Eighty (78.4\%) of these cultures were confirmed as $S$. aureus using the STAPHYtest 24 and Phoenix identification systems combined. Thus, the overall positive culture rate of $S$. aureus was $9.8 \%$ (80 positive samples out of 815 samples tested). The $S$. aureus isolates were recovered from all but 3 (IV, VI, and IX) dairy farms under the study, with 1 farm (XII) accounting for over one-third of the isolates $(27 / 80)$.

All 80 S. aureus isolates were subjected to antimicrobial susceptibility testing, the results of which are presented in Figure 1. All of the isolates were fully susceptible to cefoxitin, chloramphenicol, gentamicin, doxycycline, ciprofloxacin, and sulfamethoxazole/ trimethoprim. Likewise, all isolates were susceptible to clindamycin and erythromycin, although the susceptibility to these drugs was intermediate in 4 and 1 isolate, respectively. None of the isolates were resistant or intermediate resistant to vancomycin, as determined by the E-test method. The MIC values for this antibiotic were within the range of 0.38 to $2 \mu \mathrm{g} / \mathrm{mL}$, with a mean value of $0.94 \mu \mathrm{g} / \mathrm{mL}$. Penicillin was the only drug whose antistaphylococcal activity varied widely, with the inhibition zone diameters spanning from 6 to $52 \mathrm{~mm}$. Forty-seven (59\%) isolates were susceptible to penicillin, whereas the remaining 33 (41\%) were penicillin resistant.

Genotyping was performed on 80 confirmed isolates of $S$. aureus. Three isolates did not yield any PCR amplification product and were, thus, excluded from the analysis. Among the remaining 77 S. aureus isolates, a total of 11 distinct $\mathrm{PCR}$ patterns $(\mathrm{A}-\mathrm{K})$ were produced (Figure 2). Of these, 7 were defined as many clusters containing $73(94.8 \%)$ isolates, whereas 4 patterns were unique and corresponded to single isolates. The 2 largest clusters representing the 2 most prevalent PCR types (D and I) contained, each, 22 isolates. Clusters designated as $\mathrm{A}, \mathrm{J}$, and $\mathrm{F}$ contained 9,8 , and 7 isolates, respectively. Cluster B comprised 3 isolates, and cluster $\mathrm{G}$ comprised 2 isolates. Regarding the distribution of specific PCR types on different dairy farms, 7 types were observed on single farms only, 2 (A and D) on 4 farms, 1 (I) on 3 farms, and another (F) on 2 farms (Table 1). Of the 11 dairy farms positive for $S$. aureus, 5 (III, V, VII, XI, and XIV) were homogeneous in terms of their bacterial population (i.e., all $S$. aureus isolates within a farm had the same PCR type). On 3 farms, isolates of 3 PCR types were observed. On another 3 farms, 2 subpopulations of $S$. aureus isolates were found, each represented by a distinct PCR type.
The antimicrobial susceptibility testing demonstrated high activity of 9 out of 10 agents evaluated against $S$. aureus mastitis isolates. The only exception was penicillin $\mathrm{G}$ to which $41 \%$ of the staphylococci were resistant. Resistance to penicillin among $S$. aureus from bovine mastitis has been encountered with increasing frequency throughout the world. However, the reported prevalence rates have varied extensively in different geographical regions. The percentage of penicillinresistant $S$. aureus isolates was found to be as high as $87 \%$ in China (Shi et al., 2010), 62\% in Turkey (Turutoglu et al., 2006), and 61\% in Estonia (Kalmus et al., 2011). A lower but still high percentage of penicillin resistance, similar to that found in the current study, was detected among $S$. aureus isolated from Argentina (40\%; Gentilini et al., 2000), France (30\%; Sakwinska et al., 2011b), and Hungary (30\%; Peles et al., 2007). Contrastingly, in Germany, the United States, and Norway, the prevalence of penicillin-resistant $S$. aureus was low, with isolation rates of 17,10 , and $6 \%$, respectively (Jørgensen et al., 2005; Anderson et al., 2006; Tenhagen et al., 2006). It is noteworthy that drug susceptibility patterns of bovine mastitis $S$. aureus determined in the current study somewhat mirrored those previously reported in Poland. According to several studies performed over the last decade, resistance to penicillin was consistently the most frequently observed phenotype, whereas resistance to other antimicrobial agents was much less common (Malinowski et al., 2002, 2008). However, when comparing more recent reports, including the present one, with those previously published from Poland, a downward shift in the frequency of drug resistance can be observed. For instance, penicillin resistance among $S$. aureus isolates has long remained at a level of around $70 \%$, which is much higher than the $41 \%$ reported herein or $24 \%$ reported in a recent study of Szweda et al. (2014). Nevertheless, $\beta$-lactams are a group of antibiotics to which mastitic staphylococci are resistant most frequently and this is linked to the fact that $\beta$-lactams rank first regarding the number of compounds commercially available and in terms of their use in the treatment of bovine mammary infections.

Several molecular methods have been used over the last years for estimating the genetic diversity and population structure of $S$. aureus isolates from bovine mastitis cases. The methods most frequently applied include pulsed-field gel electrophoresis (PFGE; Jørgensen et al., 2005; Anderson et al., 2006; Peles et al., 2007; Rabello et al., 2007; Ikawaty et al., 2009; Kozytska et al., 2010), multilocus sequence typing (Jørgensen et al., 2005; Smith et al., 2005; Rabello et al., 2007; Ikawaty et al., 2009; Kozytska et al., 2010), coagulase gene (coa) typing (Schlegelová et al., 2003; Karahan and Cetinkaya, 2007; Dastmalchi Saei et al., 2009), and 


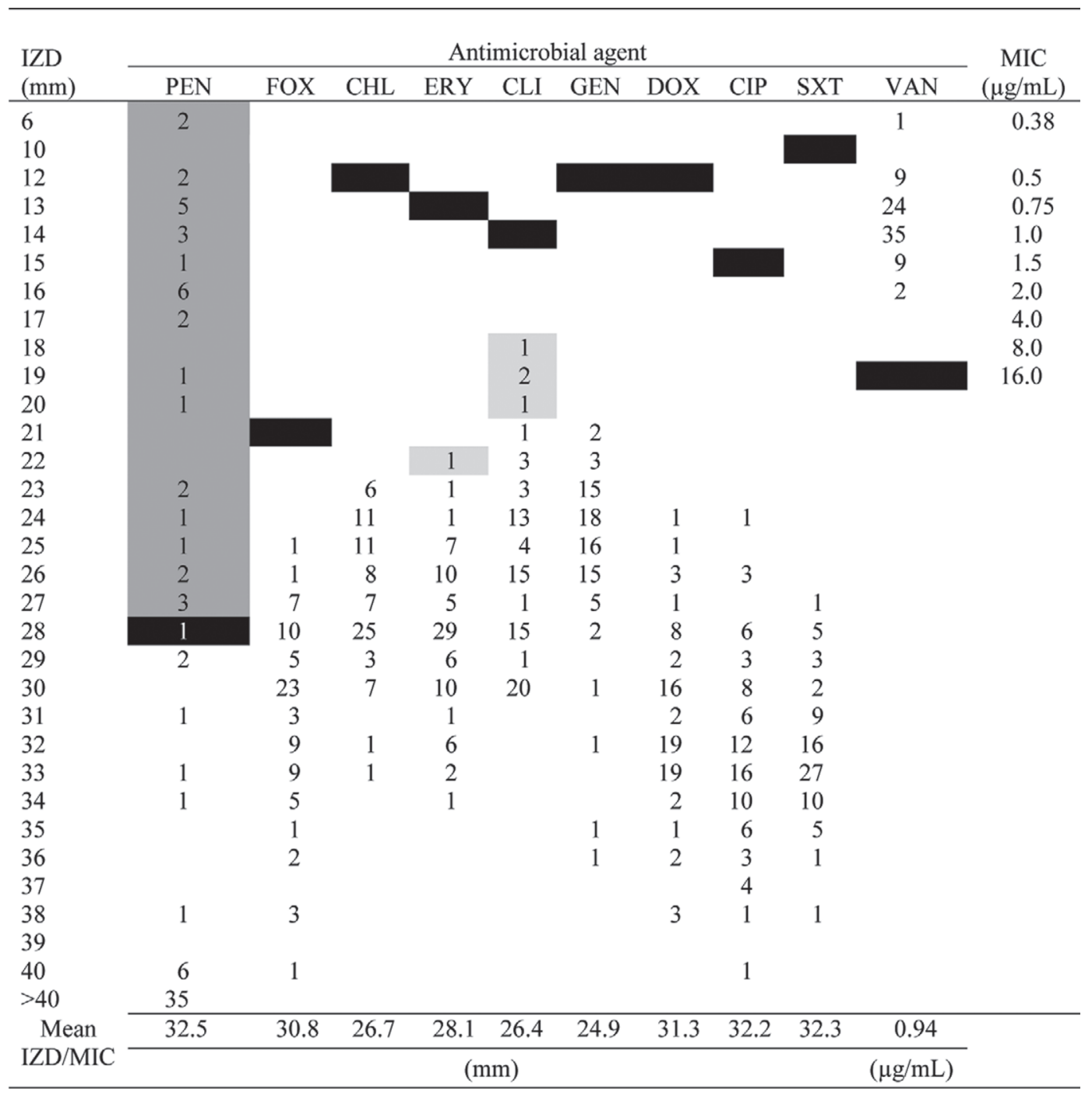

Figure 1. Distribution of inhibition zone diameter values (or MIC values) of different antimicrobial agents for the 80 Staphylococcus aureus strains evaluated in the study. The MIC values refer only to vancomycin (VAN). The black squares represent resistant zone diameter breakpoints. The squares shaded in dark and light gray represent zone diameters in the resistant and intermediate range, respectively. IZD $=$ inhibition zone diameter; PEN = penicillin; FOX = cefoxitin; $\mathrm{CHL}=$ chloramphenicol; $\mathrm{ERY}=$ erythromycin; $\mathrm{CLI}=$ clindamycin; GEN $=$ gentamicin; $\mathrm{DOX}=$ doxycycline; CIP = ciprofloxacin; $\mathrm{SXT}=$ trimethoprim/sulfamethoxazole.

spa gene typing (Ikawaty et al., 2009; Sakwinska et al., 2011b). In the current study, a triplex PCR method, targeting 3 heterogeneous loci, namely the spa gene, the coa gene, and the hypervariable region adjacent to the mecA gene, was used. This method, designed as an alternative to the PFGE gold standard, provides an excellent tool for rapid and cost-effective typing of S. aureus in outbreak settings (Strandén et al., 2003; Sabat et al., 2006). The genotyping results showed a low level of genetic differentiation among the $S$. aureus isolates studied. With only 11 PCR types obtained, the overall genetic diversity rate, calculated as the number of different patterns divided by the number of isolates, was $14.3 \%$. This was very similar to what was observed in other studies that exploited the polymorphism of the coa gene. The genetic diversity rates in those studies ranged from 12.5 to $15.5 \%$ (Schlegelová et al., 2003; Karahan and Cetinkaya, 2007; Dastmalchi Saei et al., 2009). Much higher genotype diversity (26.4-47\%) was demonstrated only when PFGE profiling was used (Peles et al., 2007; Rabello et al., 2007; Ikawaty et al., 2009; Kozytska et al., 2010). However, regardless of the genetic variation for the entire population, the genotype diversity for the individual farms was usually very low. In other words, a very limited number of genotypes existed among $S$. aureus isolates within farms. Moreover, on farms where 2 or more $S$. aureus genotypes were circulating, usually one clearly predominated 


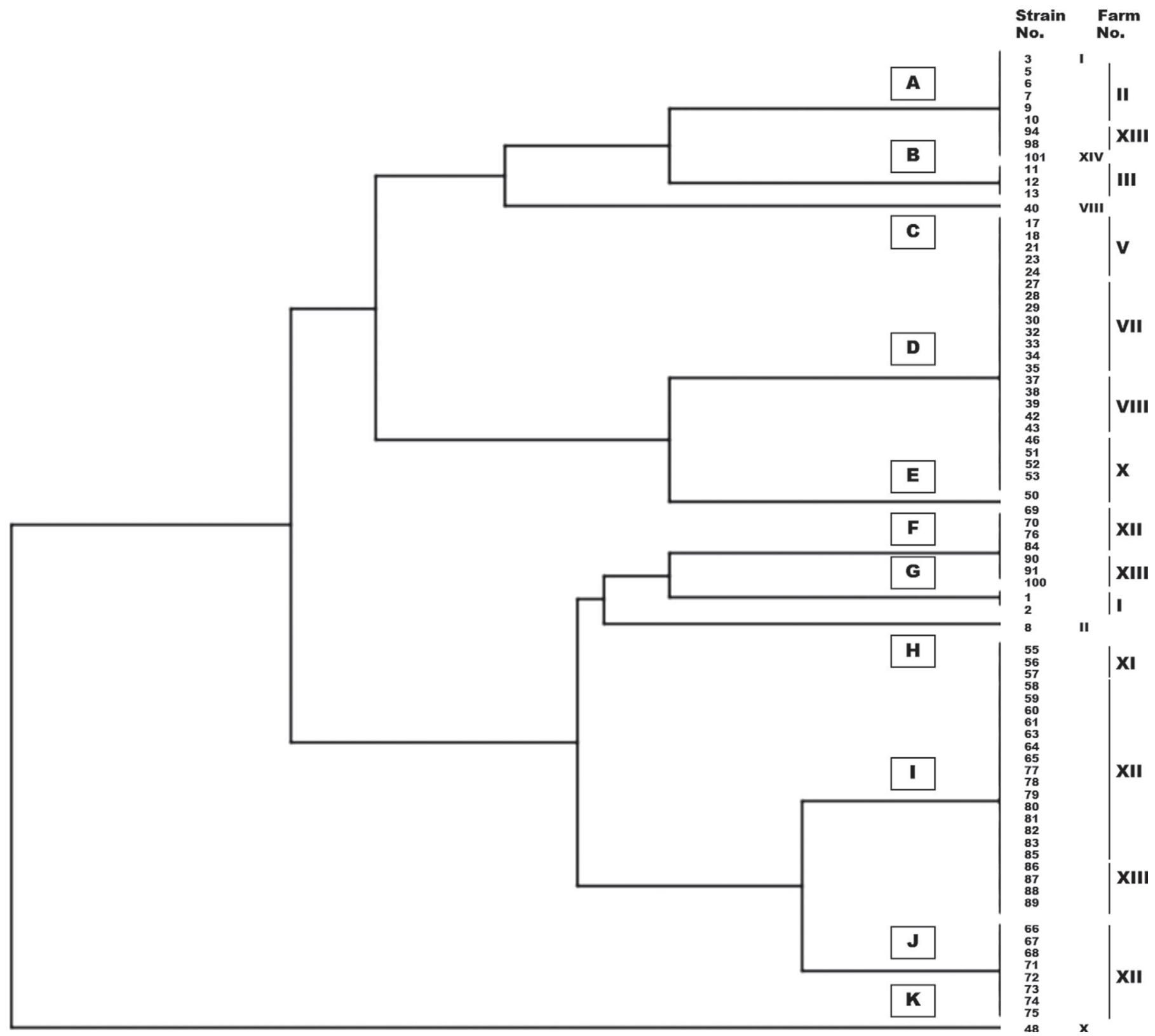

Figure 2. A dendrogram of triplex PCR patterns of the 77 Staphylococcus aureus isolates recovered from bovine mastitis cases. The boxed letters $(\mathrm{A}-\mathrm{K})$ designate clusters represented by different triplex PCR types.

over the others (Schlegelová et al., 2003; Rabello et al., 2007; Gao et al., 2012). In line with these observations are the findings in the present study. Here, up to 3 $S$. aureus genotypes were found on each of the farms evaluated. The majority of the genotypes $(7 / 11 ; 63.6 \%)$ were unique to a particular dairy farm. Four genotypes were distributed among 2 to 4 different farms, some of which were located within an over-100-km radius of each other. The finding of the same genotype in such distant locations can be explained in various ways. First, the interregional herd movement might be the cause. Second, the pathogen can be spread with humans as carriers. This hypothesis is likely, as cases of both human-to-cow and cow-to-human transmission have recently been evidenced (Sakwinska et al., 2011a). Finally, the retrieval of identical genotypes from widely separated locations can be explained by a phenomenon of homoplasy; that is, independent acquisition of similar structures without common ancestors. Another possible reason may relate to the insufficient discriminatory power of the genotyping method applied. Of particular notice are $2 S$. aureus genotypes, designated as D 
Table 1. Distribution of Staphylococcus aureus triplex PCR types among dairy farms evaluated in this study ${ }^{1}$

\begin{tabular}{|c|c|c|c|c|c|c|c|c|c|c|c|c|c|}
\hline Item & \multicolumn{11}{|c|}{ No. of $S$. aureus isolates with a PCR type: } & \multicolumn{2}{|c|}{ No. of isolates } \\
\hline \multicolumn{14}{|l|}{ Dairy farm } \\
\hline II & 5 & & & & & & & 1 & & & & 6 & 1 \\
\hline III & & 3 & & & & & & & & & & 3 & 0 \\
\hline IV & & & & & & & & & & & & 0 & 3 \\
\hline VIII & & & 1 & 5 & & & & & & & & 6 & 1 \\
\hline IX & & & & & & & & & & & & 0 & 2 \\
\hline $\mathrm{X}$ & & & & 4 & 1 & & & & & & 1 & 6 & 2 \\
\hline XI & & & & & & & & & 3 & & & 3 & 1 \\
\hline XII & & & & & & 4 & & & 15 & 8 & & 27 & 1 \\
\hline XIII & 2 & & & & & 3 & & & 4 & & & $9+2$ & 4 \\
\hline
\end{tabular}

${ }^{1}$ Underlined numbers indicate isolates for which no PCR product was obtained.

and I, both accounting for $57 \%$ of the isolates tested. Genotype D was found on 4 dairy farms located in the same municipality, suggesting transmission of this type between the farms. Here, the direction of transmission of the staphylococcal infection might have followed the chronology of strain isolation (Table 2).

Genotype I was demonstrated on 3 farms, far away from each other, making the transmission less plausible. On those farms, where the genotypes D and I cooccurred with 1 or 2 additional genotypes, the former were the most abundant. The predominance of these 2 genotypes can be associated with their increased virulence, resistance to the host immune response, and enhanced potential for persistence and replication in the host mammary tissue. Interestingly, half $(12 / 22)$ of the genotype I isolates were resistant to penicillin.

Table 2. Distribution of 4 triplex PCR types that occurred on more than 1 farm and the dates of isolation of Staphylococcus aureus strains harboring those types

\begin{tabular}{lcll}
\hline $\begin{array}{l}\text { PCR } \\
\text { type }\end{array}$ & $\begin{array}{c}\text { No. of } \\
\text { isolates }\end{array}$ & $\begin{array}{l}\text { Dairy } \\
\text { farm }\end{array}$ & $\begin{array}{l}\text { Date of } \\
\text { isolation }\end{array}$ \\
\hline A & 1 & I & May 2007 \\
& 5 & II & April 2008 \\
& 2 & XIII & May 2009 \\
D & 1 & XIV & May 2007 \\
& 5 & V & August 2008 \\
& 8 & VII & October 2008 \\
F & 5 & VIII & October 2008 \\
& 4 & X & March 2009 \\
I & 4 & XII & April 2009 \\
& 3 & XIII & May 2009 \\
& 3 & XI & March 2009 \\
& 15 & XII & April 2009 \\
& 4 & XIII & May 2009 \\
\hline
\end{tabular}

These isolates amounted to one-third $(12 / 33)$ of all penicillin-resistant $S$. aureus isolates from this study.

In conclusion, the present work showed the population structure of $S$. aureus isolates from mastitis-affected dairy cows to be highly clonal, with either exclusively one or one predominant genotype circulating within a farm. The observed similarities in the genotype composition of $S$. aureus populations from geographically distant farms underscore the importance of interfarm transmission of $S$. aureus in Poland. This, in turn, argues for the establishment of a nationwide surveillance program for bovine mastitis due to $S$. aureus. Of the antimicrobial agents, whose in vitro activity against S. aureus was evaluated, only testing for penicillin G produced drug resistance. More than $40 \%$ of the bacterial isolates were penicillin resistant, indicating a limited role of that drug and other $\beta$-lactamase-sensitive $\beta$-lactams in the treatment of bovine mastitis caused by $S$. aureus.

\section{ACKNOWLEDGMENTS}

This work was supported in part by the grant N N401 017740 from the Polish Ministry of Science and Higher Education (Warsaw, Poland).

\section{REFERENCES}

Anderson, K. L., R. L. Lyman, S. M. Bodeis-Jones, and D. G. White. 2006. Genetic diversity and antimicrobial susceptibility profiles among mastitis-causing Staphylococcus aureus isolated from bovine milk samples. Am. J. Vet. Res. 67:1185-1191.

Botrel, M.-A., M. Haenni, E. Morignat, P. Sulpice, J.-Y. Madec, and D. Calavas. 2010. Distribution and antimicrobial resistance of clinical and subclinical mastitis pathogens in dairy cows in RhôneAlpes, France. Foodborne Pathog. Dis. 7:479-487. 
CLSI (Clinical Laboratory Standards Institute). 2012. Performance standards for antimicrobial susceptibility testing. 22nd informational Supplement M100-S22, vol. 32 no. 3. CLSI, Wayne, PA.

Dastmalchi Saei, H., M. Ahmadi, K. Mardani, and R. A. Batavani. 2009. Molecular typing of Staphylococcus aureus isolated from bovine mastitis based on polymorphism of the coagulase gene in the north west of Iran. Vet. Microbiol. 137:202-206.

Gao, J., M. Ferreri, F. Yu, X. Liu, L. Chen, J. Su, and B. Han. 2012. Molecular types and antibiotic resistance of Staphylococcus aureus isolates from bovine mastitis in a single herd in China. Vet. J. 192:550-552.

Gentilini, E., G. Denamiel, P. Llorente, S. Godaly, M. Rebuelto, and O. DeGregorio. 2000. Antimicrobial susceptibility of Staphylococcus aureus isolated from bovine mastitis in Argentina. J. Dairy Sci. 83:1224-1227.

Huijps, K., T. J. Lam, and H. Hogeveen. 2008. Costs of mastitis: Facts and perception. J. Dairy Res. 75:113-120.

Ikawaty, R., E. C. Brouwer, M. D. Jansen, E. van Duijkeren, D. Mevius, J. Verhoef, and A. C. Fluit. 2009. Characterization of Dutch Staphylococcus aureus from bovine mastitis using a multiple locus variable number tandem repeat analysis. Vet. Microbiol. 136:277-284

Jørgensen, H. J., T. Mørk, D. A. Caugant, A. Kearns, and L. M. Rørvik. 2005. Genetic variation among Staphylococcus aureus strains from Norwegian bulk milk. Appl. Environ. Microbiol. 71:83528361

Kalmus, P., B. Aasmäe, A. Kärssin, T. Orro, and K. Kask. 2011. Udder pathogens and their resistance to antimicrobial agents in dairy cows in Estonia. Acta Vet. Scand. 53:4.

Karahan, M., and B. Cetinkaya. 2007. Coagulase gene polymorphisms detected by PCR in Staphylococcus aureus isolated from subclinical bovine mastitis in Turkey. Vet. J. 174:428-431.

Kozytska, S., D. Stauß, M.-C. Pawlik, S. Hensen, M. Eckart, W Ziebuhr, W. Witte, and K. Ohlsen. 2010. Identification of specific genes in Staphylococcus aureus strains associated with bovine mastitis. Vet. Microbiol. 145:360-365.

Krukowski, H., A. Lisowski, P. Rózański, and A. Skórka. 2006. Yeasts and algae isolated from cows with mastitis in the south-eastern part of Poland. Pol. J. Vet. Sci. 9:181-184.

Malinowski, E., A. Kłossowska, M. Kaczmarowski, H. Lassa, and K. Kuźma. 2002. Antimicrobial susceptibility of staphylococci isolated from affected with mastitis cows. Bull. Vet. Inst. Pulawy 46:289-294.

Malinowski, E., H. Lassa, A. Kłossowska, S. Smulski, H. Markiewicz, and M. Kaczmarowski. 2006. Etiological agents of dairy cows' mastitis in western part of Poland. Pol. J. Vet. Sci. 9:191-194.

Malinowski, E., H. Lassa, S. Smulski, A. Kłossowska, and M. Kaczmarowski. 2008. Antimicrobial susceptibility of bacteria with mastitis in 2006-2007. Bull. Vet. Inst. Pulawy 52:565-572.

Miranda-Morales, R. E., V. Rojas-Trejo, R. Segura-Candelas, E. M. Carrillo-Casas, M. G. Sánchez-Gonzalez, R. S. Castor, and F. J. Trigo-Tavera. 2008. Prevalence of pathogens associated with bovine mastitis in bulk tank milk in Mexico. Ann. N. Y. Acad. Sci. 1149:300-302.

Moroni, P., G. Pisoni, M. Antonini, R. Villa, P. Boettcher, and S. Carli. 2006. Short communication: Antimicrobial drug susceptibility of Staphylococcus aureus from subclinical bovine mastitis in Italy. J. Dairy Sci. 89:2973-2976.

Pavel, A. B., and C. I. Vasile. 2012. PyElph-A software tool for gel images analysis and phylogenetics. BMC Bioinformatics 13:9.
Peles, F., M. Wagner, L. Varga, I. Hein, P. Rieck, K. Gutser, P. Keresztúri, G. Kardos, I. Turcsányi, B. Béri, and A. Szabó. 2007. Characterization of Staphylococcus aureus strains isolated from bovine milk in Hungary. Int. J. Food Microbiol. 118:186-193.

Persson, Y., A.-K. J. Nyman, and U. Grönlund-Andersson. 2011. Etiology and antimicrobial susceptibility of udder pathogens from cases of subclinical mastitis in dairy cows in Sweden. Acta Vet. Scand. 53:36.

Rabello, R. F., B. M. Moreira, R. M. Lopes, L. M. Teixeira, L. W. Riley, and A. C. Castro. 2007. Multilocus sequence typing of Staphylococcus aureus isolates recovered from cows with mastitis in Brazilian dairy herds. J. Med. Microbiol. 56:1505-1511.

Sabat, A., N. Malachowa, J. Międzobrodzki, and W. Hryniewicz. 2006. Comparison of PCR-based methods for typing Staphylococcus aureus isolates. J. Clin. Microbiol. 44:3804-3807.

Sakwinska, O., M. Giddey, M. Moreillon, D. Morisset, A. Waldvogel, and P. Moreillon. 2011a. Staphylococcus aureus host range and human-bovine host shift. Appl. Environ. Microbiol. 77:5908-5915.

Sakwinska, O., D. Morisset, J.-Y. Madec, A. Waldvogel, P. Moreillon, and M. Haenni. 2011b. Link between genotype and antimicrobial resistance in bovine mastitis-related Staphylococcus aureus strains, determined by comparing Swiss and French isolates from the Rhône Valley. Appl. Environ. Microbiol. 77:3428-3432.

Schlegelová, J., M. Dendis, J. Benedík, V. Babák, and D. Ryšánek. 2003. Staphylococcus aureus isolates from dairy cows and humans on a farm differ in coagulase genotype. Vet. Microbiol. 92:327334.

Shi, D., Y. Hao, A. Zhang, B. Wulan, and X. Fan. 2010. Antimicrobial resistance of Staphylococcus aureus isolated from bovine mastitis in China. Transbound. Emerg. Dis. 57:221-224.

Smith, E. M., L. E. Green, G. F. Medley, H. E. Bird, L. K. Fox, Y H. Schukken, J. V. Kruze, A. J. Bradley, R. N. Zadoks, and C. G. Dowson. 2005. Multilocus sequence typing of intercontinental bovine Staphylococcus aureus isolates. J. Clin. Microbiol. 43:47374743.

Strandén, A., R. Frei, and A. F. Widmer. 2003. Molecular typing of methicillin-resistant Staphylococcus aureus: Can PCR replace pulsed-field gel electrophoresis? J. Clin. Microbiol. 41:3181-3186.

Szweda, P., M. Schielmann, A. Frankowska, B. Kot, and M. Zalewska. 2014. Antibiotic resistance in Staphylococcus aureus strains isolated from cows with mastitis in the eastern Poland and analysis of susceptibility of resistant strains to alternative non-antibiotic agents: Lysostaphin, nisin and polymyxin B. J. Vet. Med. Sci. $76: 355-362$

Tenhagen, B. A., I. Hansen, A. Reinecke, and W. Heuwieser. 2009 Prevalence of pathogens in milk samples of dairy cows with clinical mastitis and in heifers at first parturition. J. Dairy Res. 76:179187.

Tenhagen, B. A., G. Köster, J. Wallmann, and W. Heuwieser. 2006. Prevalence of mastitis pathogens and their resistance against antimicrobial agents in dairy cows in Brandenburg, Germany. J. Dairy Sci. 89:2542-2551.

Turutoglu, H., S. Ercelik, and D. Ozturk. 2006. Antibiotic resistance of Staphylococcus aureus and coagulase-negative staphylococci isolated from bovine mastitis. Bull. Vet. Inst. Pulawy 50:41-45.

Zadoks, R. N., J. R. Middleton, S. McDougall, J. Katholm, and Y. H. Schukken. 2011. Molecular epidemiology of mastitis pathogens of dairy cattle and comparative relevance to humans. J. Mammary Gland Biol. Neoplasia 16:357-372. 